\title{
Modelling stormwater treatment systems using MUSIC: accuracy
}

\begin{abstract}
Urban/rural stormwater quantity modelling has been well-researched and has achieved sufficient accuracy benchmark. However, modelling stormwater runoff quality (i.e. pollutants transport associated with stormwater) are relatively difficult and largely depends on catchment characteristics/land-uses; these can be estimated with acceptable accuracy provided pollutants transport equations are established through extensive field measurements. To ensure ecologically sustainable development, several stormwater treatment systems have been proposed. Model of Urban Stormwater Improvement Conceptualisation (MUSIC) developed and widely used in Australia, estimates pollutant transport from catchments and stormwater treatment through different systems. This paper presents a study on the accuracy of MUSIC estimations for different stormwater treatment options used in Australia and abroad. Data on several field measurements on different constructed stormwater treatment systems (bioretention, grass swale and porus pavement) in Australia, Sweden, New Zealand and Scotland was collected from literatures. The experimental results were compared with MUSIC's estimations under similar conditions. In general, it has been found that MUSIC can simulate flow conditions with good accuracy, however MUSIC's predictions on the removal efficiencies of Total Suspended Solids (TSS), Total Phosphorus (TP) and Total Nitrogen (TN) are varying. Potential reasons for these discrepancies are discussed. Also, a summary table showing MUSIC's overall capability on simulating stormwater treatment efficiencies for different treatment measures has been presented.
\end{abstract}

Keyword: Stormwater pollutants; Stormwater treatment; MUSIC; TSS; TP and TN 